\title{
Solitary Nodular Invasive Mucinous Adenocarcinoma of the Lung: Imaging Diagnosis Using the Morphologic- Metabolic Dissociation Sign
}

\author{
Min Jae Cha, $M D^{1,2}$, Kyung Soo Lee, $M D^{1}$, Tae Jung Kim, MD ${ }^{1}$, Hyun Su Kim, MD ${ }^{1}$, Tae Sung Kim, MD ${ }^{1}$, \\ Myung Jin Chung, $M D^{1}$, Byung Tae Kim, $M D^{3}$, Yang Soo Kim, $M D^{2}$ \\ ${ }^{1}$ Department of Radiology and Center for Imaging Science, Samsung Medical Center, Sungkyunkwan University School of Medicine, Seoul, Korea; \\ ${ }^{2}$ Department of Radiology, Chung-Ang University Hospital, Chung-Ang University College of Medicine, Seoul, Korea; ${ }^{3}$ Department of Nuclear \\ Medicine, Samsung Medical Center, Sungkyunkwan University School of Medicine, Seoul, Korea
}

Objective: To evaluate the efficacy of the morphologic-metabolic (M-M) dissociation sign based on computed tomography (CT) and fluorine-18-fluorodeoxyglucose positron emission tomography (PET)/CT in discriminating invasive mucinous adenocarcinoma (IMA) from invasive non-mucinous adenocarcinomas (ADCs) of the lung.

Materials and Methods: The Institutional Review Board approved this retrospective study. Among surgically resected solitary pulmonary nodule (SPN)-type ADCs ( $<3 \mathrm{~cm}$ in diameter), 35 patients with IMAs and 329 with invasive non-mucinous ADCs were included. Morphologic malignancy was established if the tumor with lobulated or spiculated margin on CT presented a tumor shadow disappearance rate of $<0.5$. The $M-M$ dissociation sign was determined when a malignant-morphologic nodule on CT showed maximum standardized uptake value (SUVmax) $<3.5$ on PET/CT.

Results: Among 35 IMAs (size: $21 \pm 7 \mathrm{~mm}$, SUVmax: $1.8 \pm 2.0$ ) and 329 invasive non-mucinous ADCs (size: $21 \pm 6 \mathrm{~mm}$, SUVmax: $4.6 \pm 4.2)$, the M-M dissociation sign was observed in $54 \%$ of IMAs $(19 / 35)$ and $10 \%$ of invasive non-mucinous ADCs $(34 / 329)(p<0.001)$. The diagnostic performance of the sign in discriminating IMA from invasive non-mucinous ADCs showed a sensitivity of $54.3 \%$ (95\% confidence interval [CI], 36.7-71.2), specificity $89.7 \%$ (95\% CI, 85.9-92.7), positive predictive value $35.8 \%$ (95\% CI, 26.5-46.5), and negative predictive value 94.9\% (95\% CI, 92.8-96.4). Multivariate analyses revealed metabolic benignity (odds ratio [OR] 2.99; 95\% CI, 1.01-8.93; $p=0.047$ ) and M-M dissociation sign (0R 6.35; $95 \%$ CI, 2.76-14.62; $p<0.001$ ) to be significant predictors of SPN-type IMAs.

Conclusion: Identification of the absence of M-M dissociation sign is an accurate indicator for excluding IMA from SPNtype lung ADCs.

Keywords: Lung; Invasive mucinous adenocarcinoma; Dissociation; CT; PET/CT

\section{INTRODUCTION}

In the 2015 World Health Organization classification as well as the 2011 classification system of International Association for the Study of Lung Cancer/American Thoracic Society/European Respiratory Society for lung adenocarcinomas (ADCs), invasive mucinous adenocarcinomas (IMAs) were classified as variants of lung $A D C s$ (1-3). In spite of the relatively low incidence of IMAs (accounting for only $2-5 \%$ of all lung $A D C s$ ), several previous studies have proved the unique characteristics of IMAs that show significant differences from invasive nonmucinous ADCs in terms of clinical, pathologic, genomic, and prognostic aspects (4-10). Histologically, IMAs are

Received June 29, 2018; accepted after revision September 18, 2018.

Corresponding author: Kyung Soo Lee, MD, Department of Radiology and Center for Imaging Science, Samsung Medical Center, Sungkyunkwan University School of Medicine, 81 Irwon-ro, Gangnam-gu, Seoul 06351, Korea.

- Tel: (822) 3410-2502 • Fax: (822) 3410-0049• E-mail: kyungs.lee@samsung.com

This is an Open Access article distributed under the terms of the Creative Commons Attribution Non-Commercial License (https:// creativecommons.org/licenses/by-nc/4.0) which permits unrestricted non-commercial use, distribution, and reproduction in any medium, provided the original work is properly cited. 
characterized by tumor cells having goblet or columnar cells with abundant intracytoplasmic mucin (4). Regarding genetic profile, IMAs show a stronger correlation with Kirsten ras (KRAS) mutation as compared to invasive non-mucinous ADCs (4-7). As for prognosis, there have been controversies about the survival outcome of IMAs. However, various prior studies demonstrated that patients with IMA showed comparable survival outcome to those with invasive nonmucinous ADCs, and IMAs are classified as an intermediategrade tumor group among all invasive $A D C s(4,8-10)$.

Although IMA is a distinct variant of lung ADCs, there is still limited information about the pre-surgical noninvasive diagnosis of IMA. Traditionally, mucinous bronchioloalveolar carcinoma (BAC) has been known to be associated with a multifocal disease or with a pneumonialike pattern on computed tomography (CT) (11). However, previous studies conducted by Lee et al. (8) and Watanabe et al. (12) demonstrated that solitary pulmonary nodule (SPN)-type IMA is much more common than pneumoniatype IMA. CT images offer little information to distinguish between nodular mucinous and non-mucinous ADCs. Meanwhile, there have been several studies indicating that mucinous BACs demonstrate relatively scant fluorine-18 $\left({ }^{18} \mathrm{~F}\right)$-flurodeoxyglucose (FDG) uptake on positron emission tomography (PET)/CT (13). We assumed that PET/CT could provide complementary metabolic information to discriminate IMA from other lung $A D C$, adding to the morphologic information of CT.

We hypothesized that $\mathrm{CT}, \mathrm{PET} / \mathrm{CT}$, and their combined features, such as morphologic-metabolic (M-M) dissociation, could provide specific finding(s) for the diagnosis of IMA. Thus, the aim of this study was to evaluate the diagnostic performance of the M-M dissociation sign, assessed on CT and $\mathrm{PET} / \mathrm{CT}$, for discriminating invasive mucinous and nonmucinous $A D C$ s of the lungs.

\section{MATERIALS AND METHODS}

The Institutional Review Board approved this retrospective study (approval 2016-12-141) and the patient consent for using clinical data was waived.

\section{Study Population}

Between September 2003 and November 2011, 942 surgically resected solitary lung $A D C s$ with pathologically confirmed negative resection margin and without evidence of residual disease on follow-up CT were identified at our institution. After exclusion of patients who had history of other malignancy and insufficient pathologic slides, all of the glass slides of resected tumors were evaluated by experienced pathologists (2). Eighty-one patients with solitary IMAs and 646 patients with solitary invasive nonmucinous ADCs were identified. Among them, 274 tumors > $3 \mathrm{~cm}$ in diameter were excluded. Eighty-nine patients were also excluded due to lack of pre-operative PET/CT. Finally, 35 patients with SPN-type IMAs ( $<3 \mathrm{~cm}$ in diameter) and 329 patients with SPN-type invasive non-mucinous ADCs, who had undergone both $\mathrm{CT}$ and $\mathrm{PET} / \mathrm{CT}$ preoperatively, were the target of this study (Fig. 1).

We screened the following clinical data including sex, age at the time of diagnosis, and treatment method from the patient medical records. Tumor, node, and metastasis (TNM) stage was determined (14). For those who underwent wedge resection, nodal staging was determined based on the preoperative $\mathrm{CT}$ and $\mathrm{PET} / \mathrm{CT}$.

942 patients underwent curative resection for SPN-type lung ADC between Sep. 2003-Nov. 2011

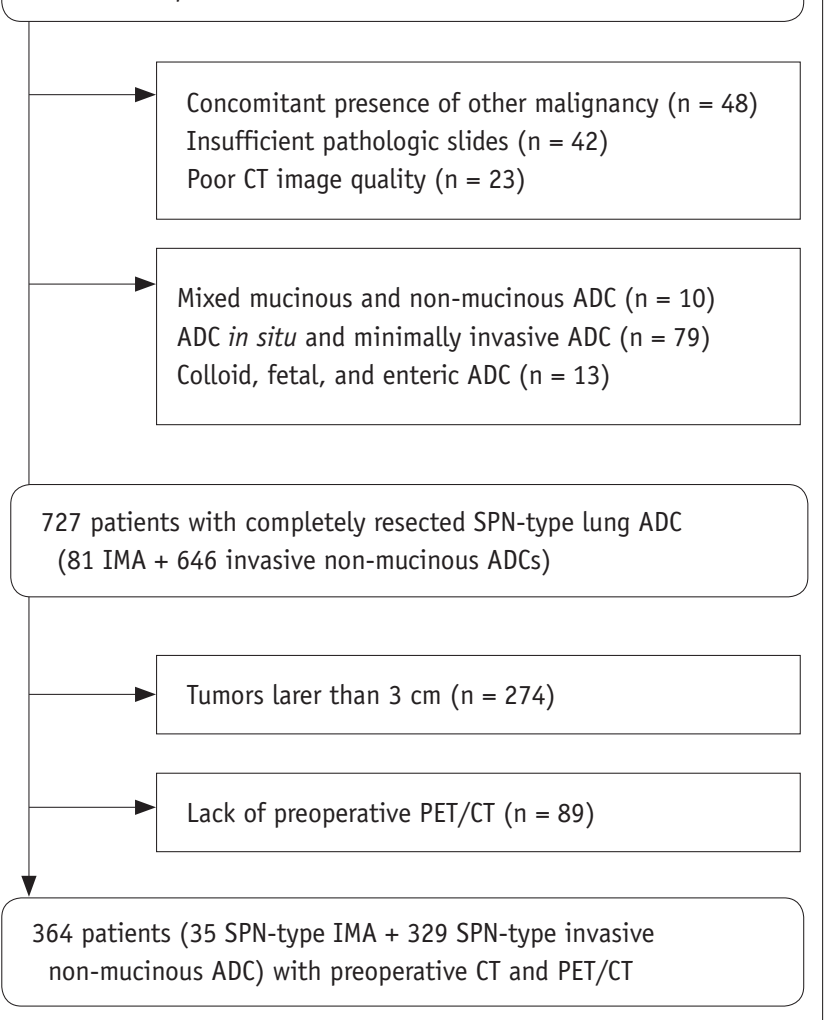

Fig. 1. Flow chart of patient selection. $A D C=$ adenocarcinoma, $C T$ = computed tomography, IMA = invasive mucinous adenocarcinoma, Nov. $=$ November, $\mathrm{PET}=$ positron emission tomography, Sep. $=$ September, SPN = solitary pulmonary nodule 


\section{Preoperative Image Acquisition and Interpretation}

Detailed parameters of chest CT and PET/CT acquisition are described in the Supplementary Materials (in the onlineonly Data Supplement). The average time interval between chest CT and PET/CT was 9.6 days (range: 0-47 days).

Two chest radiologists (with 7 and 31 years of experience in chest imaging interpretation, respectively), unaware of the clinical and PET/CT findings and histologic diagnoses, independently assessed the CT scans retrospectively. All CT scans were evaluated in terms of margin and tumor shadow disappearance rate (TDR) on non-contrast enhanced images. Tumor margin was classified into two categories: 1) smooth margin and 2) lobulated or spiculated margin. A nodule with lobulated or spiculated margin can show distortion of adjacent pulmonary parenchyma and vessels, often described as having a sunburst appearance (15). Disagreements regarding tumor margin between the two observers were solved by consensus. For acquisition of TDR, the observers measured the maximum dimension of the tumors (maxD) and the largest dimension perpendicular (perD) to the maximum axis using both the lung (width, $1500 \mathrm{HU}$; level, $-700 \mathrm{HU}$ ) and mediastinal (width, $400 \mathrm{HU}$; level, $20 \mathrm{HU}$ ) window settings on axial scans. TDR was defined as follows $(16,17)$.

$\operatorname{TDR}(\%)=1-\frac{\operatorname{maxD} \times \text { perD on mediastinal window images }}{\operatorname{maxD} \times \text { perD on lung window images }} \times 100$

Discrepancies of TDR between the two observers were resolved by averaging their measurements.

For ${ }^{18} \mathrm{~F}-\mathrm{FDG} \mathrm{PET} / \mathrm{CT}$ evaluation, a nuclear medicine physician (with 15 years of experience in PET/CT interpretation) unaware of the clinical and pathologic results evaluated the PET/CT images. Regions of interest (ROIs) were placed over the most intense area of FDG uptake in the primary tumor. FDG uptake within the ROIs was analyzed to determine the maximum standardized uptake value (SUVmax).

Morphologic malignancy was established when the TDR was $<0.5$ for a nodule showing either lobulated or spiculated margin on CT. The determination of TDR cutoff value was based on the previous studies, in which lung $A D C s$, particularly those $\leq 2 \mathrm{~cm}$ in diameter, with > $50 \%$ ground-glass opacity (GG0) component showed good survival outcome with few nodal metastases $(18,19)$. We adopted TDR as an imaging parameter to reflect the extent of GGO within the tumor. Metabolic malignancy was defined at SUVmax $\geq 3.5$ on PET/CT. The M-M dissociation sign was determined when a malignant-morphologic nodule on CT showed SUVmax $<3.5$ on PET/CT. The threshold value of 3.5 was chosen according to the receiver operating curve (ROC) analysis results for discriminating benign from malignant nodules at our institution (20).

\section{Statistical Analysis}

Student's $t$ test, the chi-square test, and Fisher's exact test were used for comparison of baseline characteristics between nodular IMA and invasive non-mucinous ADC. The Kruskal-Wallis test was used to compare imaging parameters according to the subtypes of invasive non-mucinous ADCs. When statistically significant differences occurred, post-test comparisons were performed by using the Mann-Whitney $\mathrm{U}$ test with Bonferroni correction. The chi-square test was applied for the comparison of the proportion of each subtype between tumors with positive and negative M-M dissociation sign. Multivariate logistic regression analyses were undertaken using stepwise forward selection to assess the clinical and imaging predictors for the presence of IMA. The variables with $p<0.10$ on univariate analysis were used as the input variables for the multivariate analysis. Kappa analysis was used for inter-rater reliability. The sensitivity, specificity, positive predictive value (PPV), and negative predictive value (NPV) in making the diagnosis of IMA were calculated. We constructed ROC curves to evaluate the diagnostic performance. The area under the ROC curve (AUC), a measure of diagnostic power, was calculated and pair wise comparisons were performed. All $p$ values $<0.05$ were considered statistically significant. All statistical analyses were performed using MedCalc (version 13.3.1.0, MedCalc Software bvba, Mariakerke, Belgium).

\section{RESULTS}

\section{Baseline Characteristics}

Detailed patient characteristics of SPN-type IMAs and invasive non-mucinous ADCs are shown in the Table 1. The proportion of IMAs among SPN-type ADCs was 9.5\% (35 of 364). Demographic factors such as age, sex, smoking history, and type of surgery did not differ significantly between patients with IMA and invasive non-mucinous $\operatorname{ADC}$ with $p=0.100, p=0.261, p=0.239$, and $p=$ 0.855 , respectively. There was no significant difference in T classification between the two groups, whereas $\mathrm{N}$ classification differed significantly between the two groups 
Table 1. Patient Characteristic of Nodular IMA and Invasive Non-Mucinous ADC

\begin{tabular}{|c|c|c|c|}
\hline Characteristics & IMA $(n=35)$ & Invasive Non-Mucinous ADC $(n=329)$ & $P$ \\
\hline Age (years), mean $\pm S D$ & $57.7 \pm 9.4$ & $60.5 \pm 9.5$ & 0.100 \\
\hline Smoking & & & 0.239 \\
\hline Never & $25(71)$ & $201(61)$ & \\
\hline Ever & $10(29)$ & $128(39)$ & \\
\hline Gender & & & 0.261 \\
\hline Male & $13(37)$ & $155(47)$ & \\
\hline Female & $22(63)$ & $174(53)$ & \\
\hline Types of surgery & & & 0.855 \\
\hline Wedge resection & $5(14)$ & $46(14)$ & \\
\hline Lobectomy & $30(86)$ & $280(85)$ & \\
\hline Pneumonectomy & $0(0)$ & $3(1)$ & \\
\hline T classification* & & & 0.984 \\
\hline T1a & $21(60)$ & $191(58)$ & \\
\hline $\mathrm{T} 1 \mathrm{~b}$ & $13(37)$ & $126(38)$ & \\
\hline $\mathrm{T} 2 \mathrm{a}$ & $1(3)$ & $11(3)$ & \\
\hline T3 & $0(0)$ & $1(0)$ & \\
\hline $\mathrm{N}$ classification & & & $0.025^{\dagger}$ \\
\hline NO & $35(100)$ & $274(83)$ & \\
\hline N1 & $0(0)$ & $40(12)$ & \\
\hline N2 & $0(0)$ & $15(5)$ & \\
\hline \multicolumn{4}{|l|}{ Morphologic evaluation } \\
\hline Size $(\mathrm{mm})$ & $21 \pm 7$ & $21 \pm 6$ & 0.757 \\
\hline TDR & $39.9 \pm 26.3$ & $50.9 \pm 33.1$ & 0.069 \\
\hline Margin & & & 0.101 \\
\hline Smooth & $6(17)$ & $100(30)$ & \\
\hline Lobulated or spiculated & $29(83)$ & $229(70)$ & \\
\hline Metabolic evaluation & & & $<0.001^{\dagger}$ \\
\hline SUVmax, mean \pm SD & $1.8 \pm 2.0$ & $4.6 \pm 4.2$ & \\
\hline Morphologic malignancy & $24(68)$ & $152(46)$ & $0.012^{\dagger}$ \\
\hline Metabolic malignancy & $5(14)$ & $170(52)$ & $<0.001^{\dagger}$ \\
\hline M-M dissociation & & & $<0.001^{\dagger}$ \\
\hline Positive & $19(54)$ & $34(10)$ & \\
\hline Negative & $16(46)$ & $295(90)$ & \\
\hline
\end{tabular}

${ }^{*}$ All tumors were less than $3 \mathrm{~cm}$ in diameter. Thirteen tumors of T2a and T3 were staged due to visceral pleural or chest wall invasion, ${ }^{\dagger} p$ value $<0.05 . A D C=$ adenocarcinoma, $I M A=$ invasive mucinous adenocarcinoma, $M-M=$ morphologic-metabolic, $S D=s t a n d a r d ~ d e v i a t i o n$, SUVmax $=$ maximum standardized uptake value, TDR = tumor shadow disappearance rate

( $p=0.984$ and $p=0.025$, respectively). None of the patients with SPN-type IMA showed lymph node metastasis.

Although there was no significant difference in tumor size between the IMAs $(21 \pm 7 \mathrm{~mm})$ and invasive non-mucinous ADCs $(21 \pm 6 \mathrm{~mm})(p=0.757)$, SUVmax was significantly higher in invasive non-mucinous ADCs $(4.6 \pm 4.2)$ than in IMAs $(1.8 \pm 2.0)(p<0.001)$ (Figs. 2-4). The TDR tended to be lower in IMAs $(39.9 \pm 26.3 \%)$ as compared to the invasive non-mucinous ADCs $(50.9 \pm 33.1 \%)$, although the difference was not statistically significant $(p=0.069)$. Seventy-one percent of nodular IMAs (25 of 35 ) and $56 \%$ of nodular invasive non-mucinous ADCs (185 of 329) showed
TDR $<0.5$. Additionally, relatively high proportion of both IMAs (83\%) and invasive non-mucinous ADCs (70\%) showed lobulated or spiculated margin on CT.

In terms of histologic subtypes of nodular invasive nonmucinous ADCs, 86 patients (26\%) had lepidic-predominant ADCs, 172 (53\%) had acinar-predominant ADCs, 24 (7\%) had papillary-predominant ADCs, $4(1 \%)$ had micropapillarypredominant ADCs, and $43(13 \%)$ had solid-predominant ADCs (Table 2). For lepidic-predominant ADCs, TDR was significantly higher and SUVmax was significantly lower as compared to those of other subtypes of non-mucinous ADCs ( $p<0.001$ for both). 


\section{Diagnostic Performance of Imaging Features}

Inter-rater reliability between two readers for the determination of morphologic malignancy was excellent (kappa value $=0.819$ ).

The rate of morphologic malignancy on CT was significantly higher in nodular IMA $(68 \%)$ than in invasive non-mucinous $\operatorname{ADC}(46 \%)$, whereas the rate of metabolic malignancy on PET/CT was significantly lower in IMA (14\%) than in invasive non-mucinous ADC (52\%) ( $p=0.012$ and $p<0.001$, respectively). Among 35 IMAs and 329 invasive non-mucinous ADCs, 19 IMAs (54\%) and 34 invasive nonmucinous ADCs (10\%) showed positive M-M dissociation

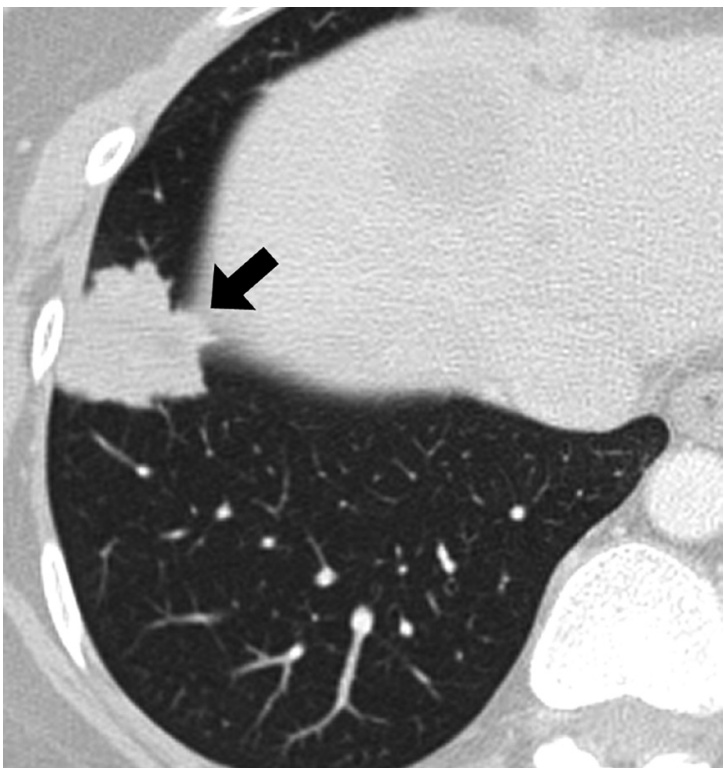

A

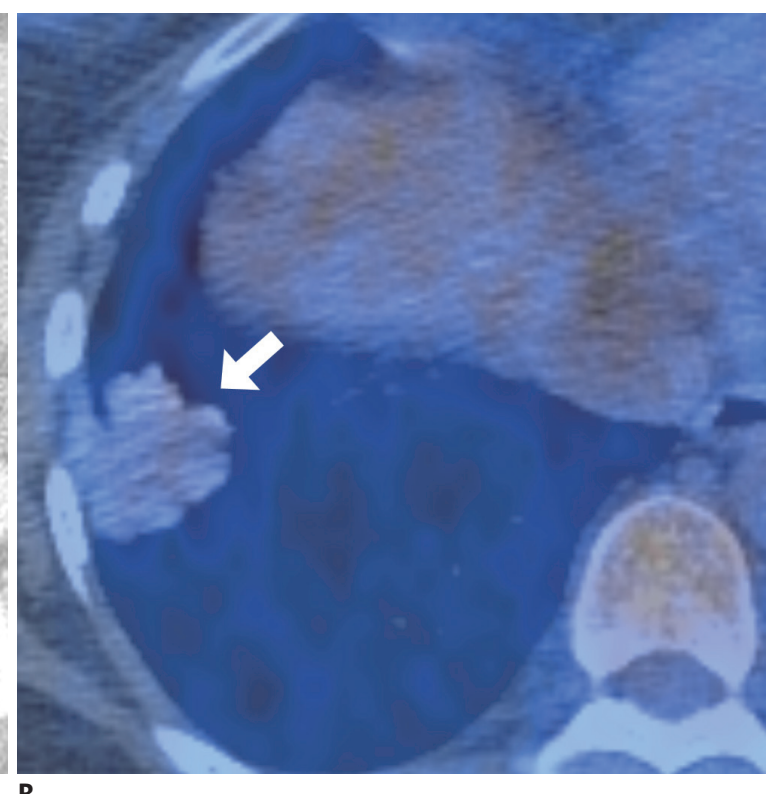

Fig. 2. IMA with positive M-M dissociation sign in 43-year-old woman.

A. Lung window image of transverse CT scan obtained at level of liver dome shows 30-mm-sized nodule with lobulated or spiculated margin (arrow) in right lower lobe $\left(\mathrm{TDR}=1.34 \%\right.$ ). B. $\mathrm{PET} / \mathrm{CT}$ image demonstrates scant ${ }^{18} \mathrm{~F}-\mathrm{FDG}$ uptake (arrow) within tumor and with SUVmax of 2.2. FDG $=$ fluorodeoxyglucose, $\mathrm{M}-\mathrm{M}=$ morphologic-metabolic, SUVmax $=$ maximum standardized uptake value, $\mathrm{TDR}=$ tumor shadow disappearance rate, ${ }^{18} \mathrm{~F}=$ fluorine-18

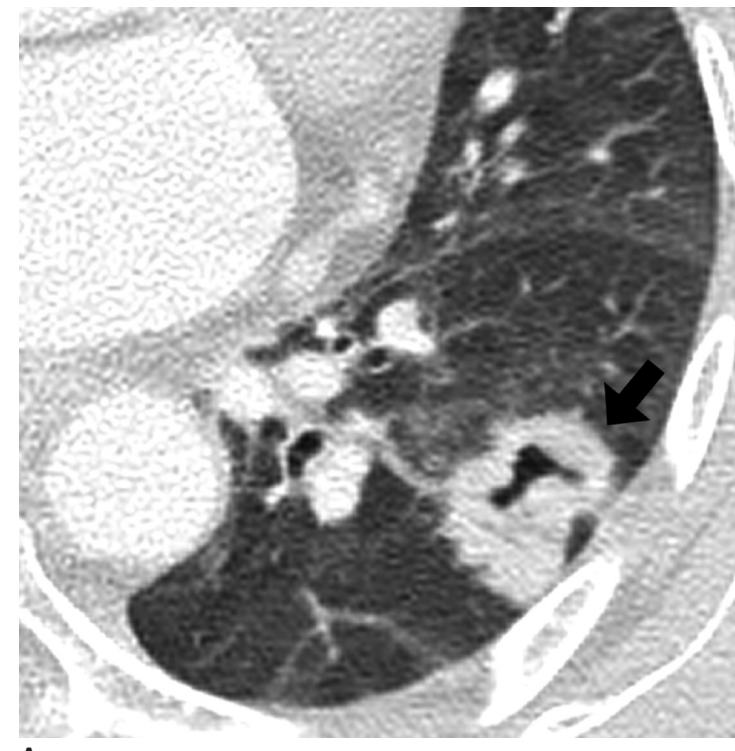

A

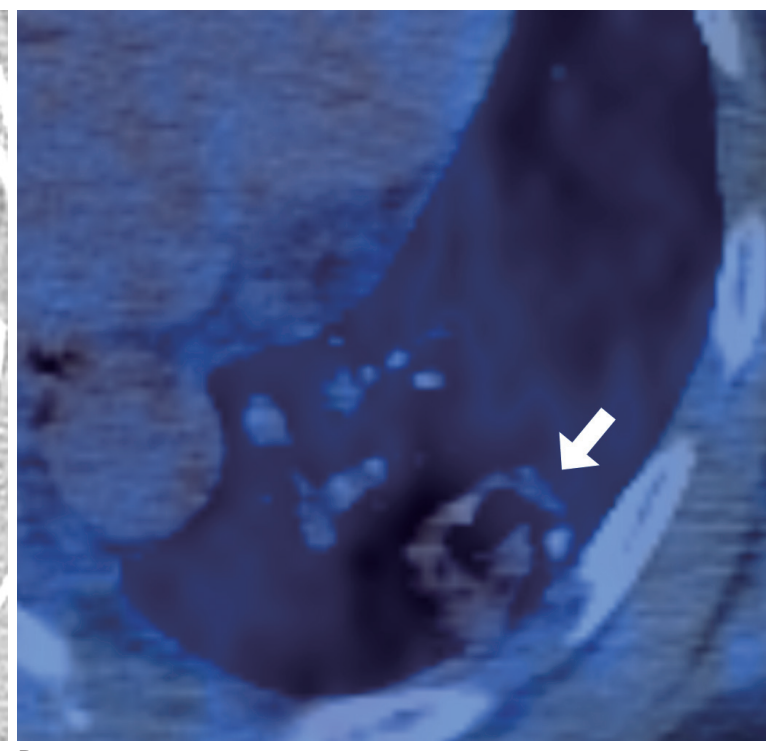

B

Fig. 3. IMA with positive M-M dissociation sign in 52-year-old woman.

A. Lung window image of transverse CT scan obtained at level of left atrium shows 27-mm-sized nodule with lobulated or spiculated margin (arrow) in left lower lobe (TDR $=28.99 \%$ ). Additionally, note internal cavitation or bubble lucency within tumor. B. PET/CT image demonstrates scant

${ }^{18}$ F-FDG uptake (arrow) within tumor and with SUVmax of 3.0. 


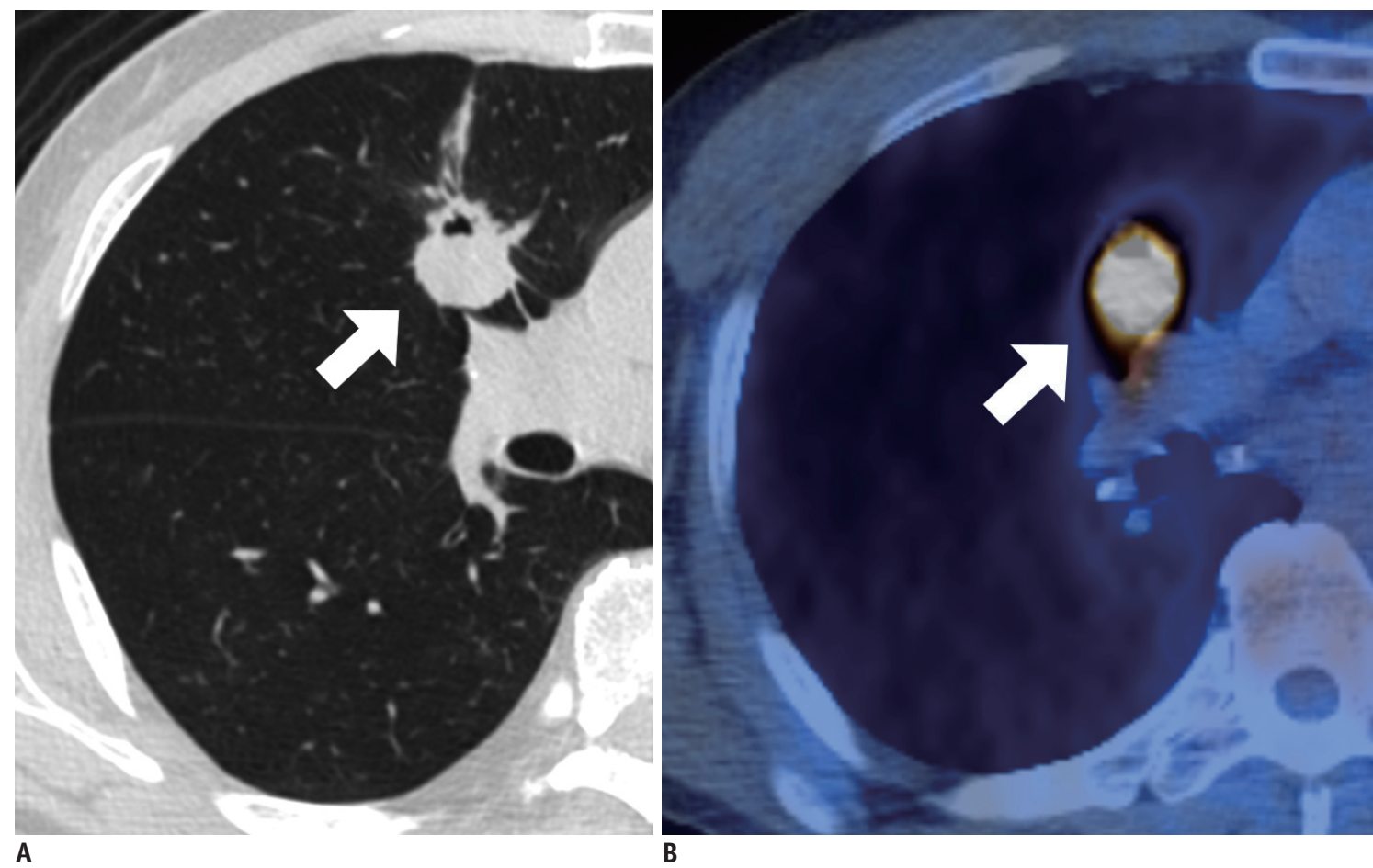

Fig. 4. Invasive non-mucinous adenocarcinoma with negative M-M dissociation sign in 60-year-old man.

A. Lung window image of transverse CT scan obtained at level of right bronchus intermedius shows 24-mm-sized lobulated and spiculated nodule (arrow) in right middle lobe $(\mathrm{TDR}=35.31 \%)$. Additionally, note internal cavitation or bubble lucency within tumor. B. PET/CT image demonstrates hot ${ }^{18} \mathrm{~F}-\mathrm{FDG}$ uptake (arrow) within tumor and with SUVmax of 12.9.

Table 2. Morphologic and Metabolic Evaluation of Nodular Invasive Non-Mucinous ADCs According to Predominant Histologic Subtype

\begin{tabular}{|c|c|c|c|c|c|}
\hline Characteristics & Lepidic $(n=86)$ & Acinar $(n=172)$ & Papillary $(n=24)$ & Micropapillary $(n=4)$ & Solid $(n=43)$ \\
\hline \multicolumn{6}{|l|}{ Morphologic evaluation } \\
\hline Size (mm) & $18 \pm 6$ & $21 \pm 6$ & $22 \pm 5$ & $25 \pm 4$ & $21 \pm 6$ \\
\hline TDR & $83.2 \pm 24.8$ & $43.1 \pm 29.2$ & $30.0 \pm 15.4$ & $26.4 \pm 18.6$ & $30.3 \pm 22.9$ \\
\hline \multicolumn{6}{|l|}{ Margin } \\
\hline Smooth & $49(57)$ & $41(24)$ & $2(8)$ & $0(0)$ & $8(19)$ \\
\hline Lobulated or spiculated & $37(43)$ & $131(76)$ & $22(92)$ & $4(100)$ & $35(81)$ \\
\hline \multicolumn{6}{|l|}{ Metabolic evaluation } \\
\hline SUVmax, mean \pm SD & $1.2 \pm 1.5$ & $5.1 \pm 3.8$ & $5.5 \pm 3.3$ & $6.3 \pm 4.3$ & $8.9 \pm 4.7$ \\
\hline Morphologic malignancy & $11(13)$ & $92(53)$ & $18(75)$ & $3(75)$ & $28(65)$ \\
\hline Metabolic malignancy & $10(12)$ & $105(61)$ & $15(63)$ & $3(75)$ & $37(86)$ \\
\hline \multicolumn{6}{|l|}{ M-M dissociation } \\
\hline Positive & $6(7)$ & $21(12)$ & $4(17)$ & $1(25)$ & $2(5)$ \\
\hline Negative & $80(93)$ & $151(88)$ & $20(83)$ & $3(75)$ & $41(95)$ \\
\hline
\end{tabular}

sign, which differed significantly between the two groups ( $p$ $<0.001$ ) (Table 1).

Regarding histologic subtypes of invasive non-mucinous $A D C s, 88 \%$ of lepidic-predominant ADCs (76 of 86 ) were metabolically benign on PET/CT with mean SUVmax of 1.2 (standard deviation, 1.5). However, the proportion of positive $\mathrm{M}-\mathrm{M}$ dissociation sign tumors was only $7 \%$ among lepidic-predominant ADCs (6 of 86), mostly due to high TDR
(83.2 \pm 24.8$)$, representing large area of GG0 component within the tumor. In terms of other subtypes such as acinar, papillary, micropapillary, and solid-predominant ADCs, they tended to show both morphologically $(58.3 \%)$ and metabolically (62.0\%) malignant features on CT and PET/CT simultaneously, resulting in relatively low rate of positive M-M dissociation sign (11.6\%) (Table 2). In the comparison between tumors having positive $(n=34)$ and negative $(n=$ 
295) M-M dissociation sign among invasive non-mucinous $A D C s$, there was no significant difference in the proportion of each subtype $(p=0.336)$.

The diagnostic performance of morphologic malignancy on CT for discriminating IMA presented sensitivity of $68.6 \%$ (24 of $35 ; 95 \%$ confidence interval [CI], 50.7-83.2), specificity $53.8 \%$ (177 of $329 ; 95 \%$ CI, 48.3-59.3), PPV $13.6 \%$ (24 of $176 ; 95 \%$ CI, 10.9-16.9), and NPV $94.2 \%$ (177 of $188 ; 95 \%$ CI, 90.7-96.4). In terms of metabolic benignity on PET/CT, the sensitivity was $85.7 \%$ (30 of 35; 95\% CI, 69.7-95.2), specificity $51.7 \%$ (170 of $329 ; 95 \%$ CI, 46.1-57.2), PPV $15.9 \%$ (30 of $189 ; 95 \%$ CI, 13.7-18.4), and NPV $97.1 \%$ (170 of $175 ; 95 \%$ CI, 93.8-98.7) in discriminating IMA from invasive non-mucinous ADCs. Finally, the diagnostic performance of the M-M dissociation sign was as follows: sensitivity $54.3 \%$ (19 of $35 ; 95 \%$ CI, 36.7-71.2), specificity $89.7 \%$ (295 of $329 ; 95 \%$ CI, 85.9-92.7), PPV $35.8 \%$ (19 of 53; 95\% CI, 26.5-46.5), and NPV 94.9\% (295 of 311; $95 \%$ CI, 92.8-96.4). In the comparison of diagnostic performance among morphologic malignancy (CT), metabolic benignity (PET/CT), and M-M dissociation sign for discriminating IMA from invasive non-mucinous ADCs, the AUC of M-M dissociation sign $(0.720 ; 95 \%$ CI, 0.616 0.823 ) was significantly greater than that of morphologic malignancy $(0.612 ; 95 \% \mathrm{CI}, 0.516-0.707)$ and metabolic benignity (0.313; 95\% CI, 0.232-0.395).

Table 3 summarizes the result of multivariate analysis for predicting IMA among SPN-type lung ADCs. N stage $(p=0.025)$, morphologic malignancy on CT $(p=0.012)$, metabolic benignity at PET $(p<0.001)$, and M-M dissociation sign $(p<0.001)$ were regarded as input variables for multivariate analysis. Multivariate analysis confirmed that metabolic benignity on PET/CT (odds ratio [OR] 2.99; 95\% CI, 1.01-8.93; $p=0.047$ ) and $M-M$ dissociation sign (OR 6.35; 95\% CI, 2.76-14.62; $p<0.001$ ) were significant predictors of nodular IMAs.

\section{DISCUSSION}

Many studies have addressed the clinico-pathologic characteristics of IMAs as an evolving disease entity.
Particularly, in terms of genetic profile, IMA is correlated with the absence of epidermal growth factor receptor (EGFR) mutations and the presence of KRAS mutations, which indicates that these tumors are unlikely to respond to EGFR-tyrosine kinase inhibitors such as gefitinib and erlotinib (4, 5, 12, 21-23). Many reports have also shown that IMA is associated with lower rates of pleural involvement, lymphatic permeation, and vascular invasion, in contrast to frequent aerogenous spread $(6-8,11,24)$. These previous studies corroborate our results that none of the SPN-type IMAs showed lymph node metastasis. In spite of these unique properties of IMA, little is known about the imaging diagnosis of IMAs.

Several recent studies revealed that SPN-type IMA is far more common than pneumonia-type IMA $(8,12)$. However, despite the relatively large number of SPN-type IMAs, there have been few reports on their morphologic characteristics on $\mathrm{CT}$ in the literature. Instead, various studies have reported interesting results on $\mathrm{PET} / \mathrm{CT}$. In a study by Chang et al. (25), mucinous BACs exhibit significantly lower peak SUVs compared to those of squamous cell carcinomas, nonmucinous $A D C s$, and other malignancies. Furthermore, Lee et al. (13) also reported that nodular IMA depicts scant FDG uptake (SUVmax $2.3 \pm 1.9$ ).

In this study, we defined the M-M dissociation sign and evaluated the diagnostic performance of this sign in discriminating SPN-type IMAs from invasive nonmucinous $A D C$. The specificity of $M-M$ dissociation sign in discriminating SPN-type IMA from invasive non-mucinous ADCs was $89.7 \%$, which was much better than that of CT (53.8\%) or PET/CT (51.7\%) alone, therefore presenting a low false positive rate. We also found that identification of the negativity of this sign is an accurate indication to exclude IMA with an NPV of $94.9 \%$. Multivariate analyses demonstrated that $M-M$ dissociation sign and benignity on PET/CT are two significant discriminators of IMA among nodular ADCs with ORs of 6.35 and 2.99, respectively. Substantially, the NPV of CT alone (94.2\%) was also comparably high and the NPV of PET/CT (97.1\%) was even higher than that of M-M dissociation sign. However, the PPV of CT (13.6\%) and PET/CT (15.9\%) alone was far lower than

Table 3. Multivariate Analyses of Clinical and Imaging Parameters for Predicting Nodular IMA

\begin{tabular}{lccrr}
\multicolumn{1}{c}{ Characteristics } & Odds Ratio & $95 \%$ CI & $P$ \\
\hline Metabolic benignity on PET/CT, SUVmax $<3.5$ & 2.99 & $1.014-8.829$ & 0.047 \\
M-M dissociation sign & 6.35 & $2.759-14.618$ & $<0.001$ \\
\hline
\end{tabular}

$\mathrm{CI}=$ confidence interval, $\mathrm{PET} / \mathrm{CT}$ = positron emission tomography/computed tomography 
that of $M-M$ dissociation sign (35.8\%), limiting the general application of CT or PET/CT alone for the diagnosis of IMA. The relatively low rate of overall sensitivity and PPV in our analysis could be partly explained by the disproportionate numbers of IMAs and invasive non-mucinous ADCs (35 versus 329). Although the number of invasive non-mucinous ADCs was markedly larger than that of IMAs, our cases might represent the real incidence of IMA among SPN-type lung ADCs.

A possible source of discrepancy between PET/CT and CT is the mucinous component within IMAs. IMAs are peculiarly prone to show lower TDR as compared to non-mucinous tumors, because of the pathologic features of tumor cells with abundant mucin. On the other hand, relatively small numbers of metabolically active cancer cells compared to the large amount of mucin can be a reason for scant FDG uptake on PET/CT in IMAs; hence, FDG uptake correlates directly with the number of cancer cells $(25,26)$. Indeed, differentiation between IMA and lepidic-predominant nonmucinous ADCs could be mostly achieved with the difference in TDR, as both of the tumor types show little FDG uptake on PET/CT. Additionally, differentiation between IMA and other non-mucinous ADCs, including acinar-, papillary-, micropapillary-, and solid-predominant ADCs, could be done based on the difference of SUVmax value because most of these tumors were morphologically malignant on CT.

In this study, we made use of marginal characteristics and TDR in determining morphologic malignancy on CT. A lobulated contour or an irregular or spiculated margin is a renowned feature suggestive of malignancy (27-30). In terms of TDR, several previous reports indicated that high TDR or a large area of GGO within a nodule is an important factor for good prognosis in lung $\operatorname{ADCs}(18,19,27,31)$. Although TDR is a known prognostic parameter of lung ADCs, we adopted TDR as a differential point of morphologic malignancy as high TDR is assumed to represent low tumor cellularity or a less aggressive tumor (32).

Our study has several limitations. First, it was limited inherently by its retrospective design, and we might have had a selection bias. Second, this study was performed in a single institution and follow-up periods were variable. Third, we only included nodular IMAs and invasive non-mucinous $A D C$ s that were $<3 \mathrm{~cm}$ in diameter. This restriction of tumor size may influence the FDG uptake on PET/CT, lowering the average value of the SUVmax. Lastly, the proportion of solid-predominant $A D C s$ among invasive non-mucinous ADCs was relatively large $(13 \%)$, which could affect the diagnostic performance of $M-M$ dissociation sign. External validation of this sign with larger study population from multiple centers can be the next step.

In conclusion, we proposed the idea of a new diagnostic condition of IMAs, the M-M dissociation sign, based on morphologic and metabolic images. We suggest that the identification of the absence of $M-M$ dissociation sign is an accurate indication to exclude IMA from SPN-type lung ADCs.

\section{Supplementary Materials}

The online-only Data Supplement is available with this article at https://doi.org/10.3348/kjr.2018.0409.

\section{Conflicts of Interest}

The authors have no financial conflicts of interest.

ORCID

Kyung Soo Lee

https://orcid.org/0000-0002-3660-5728

Min Jae Cha

https://orcid.org/0000-0001-6358-8081

\section{REFERENCES}

1. Travis WD, Brambilla E, Noguchi M, Nicholson AG, Geisinger KR, Yatabe $Y$, et al. International Association for the Study of Lung Cancer/American Thoracic Society/European Respiratory Society international multidisciplinary classification of lung adenocarcinoma. J Thorac Oncol 2011;6:244-285

2. Travis WD, Brambilla E, Burke AP, Marx A, Nicholson AG. Introduction to The 2015 World Health Organization Classification of tumors of the lung, pleura, thymus, and heart. J Thorac Oncol 2015;10:1240-1242

3. Travis WD, Brambilla E, Burke AP, Marx A, Nicholson AG. WHO classification of tumours of the lung, pleura, thymus and heart, 4th ed. Lyon: IARC Publications, 2015

4. Shim HS, Kenudson M, Zheng Z, Liebers M, Cha YJ, Hoang Ho $Q$, et al. Unique genetic and survival characteristics of invasive mucinous adenocarcinoma of the lung. J Thorac Oncol 2015; 10:1156-1162

5. Finberg KE, Sequist LV, Joshi VA, Muzikansky A, Miller JM, Han $M$, et al. Mucinous differentiation correlates with absence of EGFR mutation and presence of KRAS mutation in lung adenocarcinomas with bronchioloalveolar features. $\mathrm{J} \mathrm{Mol}$ Diagn 2007;9:320-326

6. Kadota K, Yeh YC, D'Angelo SP, Moreira AL, Kuk D, Sima CS, et al. Associations between mutations and histologic patterns of mucin in lung adenocarcinoma: invasive mucinous pattern and extracellular mucin are associated with KRAS mutation. Am J Surg Pathol 2014;38:1118-1127 
Invasive Mucinous ADCs of the Lung: Diagnosis with M-M Dissociation Sign

7. Duruisseaux M, Antoine M, Rabbe N, Poulot V, Fleury-Feith J, Vieira $T$, et al. The impact of intracytoplasmic mucin in lung adenocarcinoma with pneumonic radiological presentation. Lung Cancer 2014;83:334-340

8. Lee HY, Cha MJ, Lee KS, Lee HY, Kwon 0J, Choi JY, et al. Prognosis in resected invasive mucinous adenocarcinomas of the lung: related factors and comparison with resected nonmucinous adenocarcinomas. J Thorac Oncol 2016;11:1064-1073

9. Yoshizawa A, Sumiyoshi S, Sonobe M, Kobayashi M, Fujimoto $M$, Kawakami F, et al. Validation of the IASLC/ATS/ERS lung adenocarcinoma classification for prognosis and association with EGFR and KRAS gene mutations: analysis of 440 Japanese patients. J Thorac Oncol 2013;8:52-61

10. Nakamura H, Saji H, Shinmyo T, Tagaya R, Kurimoto N, Koizumi $\mathrm{H}$, et al. Close association of IASLC/ATS/ERS lung adenocarcinoma subtypes with glucose-uptake in positron emission tomography. Lung Cancer 2015;87:28-33

11. Casali C, Rossi G, Marchioni A, Sartori G, Maselli F, Longo L, et al. A single institution-based retrospective study of surgically treated bronchioloalveolar adenocarcinoma of the lung: clinicopathologic analysis, molecular features, and possible pitfalls in routine practice. J Thorac Oncol 2010;5:830-836

12. Watanabe H, Saito H, Yokose T, Sakuma Y, Murakami S, Kondo $\mathrm{T}$, et al. Relation between thin-section computed tomography and clinical findings of mucinous adenocarcinoma. Ann Thorac Surg 2015;99:975-981

13. Lee HY, Lee KS, Han J, Kim BT, Cho YS, Shim YM, et al. Mucinous versus nonmucinous solitary pulmonary nodular bronchioloalveolar carcinoma: CT and FDG PET findings and pathologic comparisons. Lung Cancer 2009;65:170-175

14. Edge SB, Compton CC. The American Joint Committee on Cancer: the 7th edition of the AJCC cancer staging manual and the future of TNM. Ann Surg Oncol 2010;17:1471-1474

15. Erasmus JJ, Connolly JE, McAdams HP, Roggli VL. Solitary pulmonary nodules: part I. Morphologic evaluation for differentiation of benign and malignant lesions. Radiographics 2000;20:43-58

16. Lee HY, Han J, Lee KS, Koo JH, Jeong SY, Kim BT, et al. Lung adenocarcinoma as a solitary pulmonary nodule: prognostic determinants of $\mathrm{CT}, \mathrm{PET}$, and histopathologic findings. Lung Cancer 2009;66:379-385

17. Okada M, Tauchi S, Iwanaga K, Mimura T, Kitamura Y, Watanabe $\mathrm{H}$, et al. Associations among bronchioloalveolar carcinoma components, positron emission tomographic and computed tomographic findings, and malignant behavior in small lung adenocarcinomas. J Thorac Cardiovasc Surg 2007;133:1448-1454

18. Aoki T, Tomoda Y, Watanabe H, Nakata H, Kasai T, Hashimoto $\mathrm{H}$, et al. Peripheral lung adenocarcinoma: correlation of thinsection CT findings with histologic prognostic factors and survival. Radiology 2001;220:803-809

19. Kim EA, Johkoh T, Lee KS, Han J, Fujimoto K, Sadohara $J$, et al. Quantification of ground-glass opacity on highresolution CT of small peripheral adenocarcinoma of the lung: pathologic and prognostic implications. AJR Am J Roentgenol 2001;177:1417-1422
20. Shim SS, Lee KS, Kim BT, Chung MJ, Lee EJ, Han J, et al. Nonsmall cell lung cancer: prospective comparison of integrated FDG PET/CT and CT alone for preoperative staging. Radiology 2005;236:1011-1019

21. Hata A, Katakami N, Fujita S, Kaji R, Imai Y, Takahashi Y, et al. Frequency of EGFR and KRAS mutations in Japanese patients with lung adenocarcinoma with features of the mucinous subtype of bronchioloalveolar carcinoma. J Thorac Oncol 2010;5:1197-1200

22. O'Neill AC, Jagannathan JP, Ramaiya NH. Evolving cancer classification in the era of personalized medicine: a primer for radiologists. Korean J Radiol 2017;18:6-17

23. Krajewski KM, Braschi-Amirfarzan M, DiPiro PJ, Jagannathan $J P$, Shinagare AB. Molecular targeted therapy in modern oncology: imaging assessment of treatment response and toxicities. Korean J Radiol 2017;18:28-41

24. Manning JT Jr, Spjut HJ, Tschen JA. Bronchioloalveolar carcinoma: the significance of two histopathologic types. Cancer 1984;54:525-534

25. Chang JM, Lee HJ, Goo JM, Lee HY, Lee JJ, Chung JK, et al. False positive and false negative FDG-PET scans in various thoracic diseases. Korean J Radiol 2006;7:57-69

26. Berger KL, Nicholson SA, Dehdashti F, Siegel BA. FDG PET evaluation of mucinous neoplasms: correlation of FDG uptake with histopathologic features. AJR Am J Roentgenol 2000;174:1005-1008

27. Cha MJ, Lee KS, Kim HS, Lee SW, Jeong CJ, Kim EY, et al. Improvement in imaging diagnosis technique and modalities for solitary pulmonary nodules: from ground-glass opacity nodules to part-solid and solid nodules. Expert Rev Respir Med 2016;10:261-278

28. Jeong YJ, Lee KS, Jeong SY, Chung MJ, Shim SS, Kim H, et al. Solitary pulmonary nodule: characterization with combined wash-in and washout features at dynamic multi-detector row CT. Radiology 2005;237:675-683

29. Jeong SY, Lee KS, Shin KM, Bae YA, Kim BT, Choe BK, et al. Efficacy of PET/CT in the characterization of solid or partly solid solitary pulmonary nodules. Lung Cancer 2008;61:186194

30. Lee KS, Yi CA, Jeong SY, Jeong YJ, Kim S, Chung MJ, et al. Solid or partly solid solitary pulmonary nodules: their characterization using contrast wash-in and morphologic features at helical CT. Chest 2007;131:1516-1525

31. Ohde Y, Nagai K, Yoshida J, Nishimura M, Takahashi K, Suzuki K, et al. The proportion of consolidation to groundglass opacity on high resolution CT is a good predictor for distinguishing the population of non-invasive peripheral adenocarcinoma. Lung Cancer 2003;42:303-310

32. Lee HY, Lee SW, Lee KS, Jeong JY, Choi JY, Kwon 0J, et al. Role of CT and PET imaging in predicting tumor recurrence and survival in patients with lung adenocarcinoma: a comparison with the International Association for the Study of Lung Cancer/American Thoracic Society/European Respiratory Society classification of lung adenocarcinoma. J Thorac Oncol 2015;10:1785-1794 